\title{
A Nuclear Magnetic Resonance and Relaxation Study of Dimethoxyborane
}

\author{
T. C. Farrar and T. Tsang \\ Institute for Materials Research, National Bureau of Standards, Washington, D.C. 20234
}

(December 10, 1968)

\begin{abstract}
Proton and boron-11 c.w. nuclear magnetic resonances have been studied in solid $\mathrm{H}^{11} \mathrm{~B}\left(\mathrm{OCD}_{3}\right)_{2}$ and $\mathrm{H}^{10} \mathrm{~B}\left(\mathrm{OCD}_{3}\right)_{2}$. For ${ }^{11} \mathrm{~B}$, only the $-\frac{1}{2} \rightarrow \frac{1}{2}$ transition, broadened by second order quadrupolar effects and by proton dipolar interaction can be seen; from the spectrum at several of frequencies, the quadrupolar coupling constant $\left|e^{2} q Q / h\right|$ was found to be $3.0 \pm 0.2 \mathrm{MHz}$ ( \pm always refers to rms errors). In $\mathrm{H}^{10} \mathrm{~B}\left(\mathrm{OCD}_{3}\right)_{2}$, the proton line shapes at 53 and $10 \mathrm{MHz}$ are considerably different; this may be interpreted as due to changes in the directions of ${ }^{10} \mathrm{~B}$ nuclear quantization. Nuclear magnetic relaxation studies have been made in the liquid phase. From the ${ }^{10} \mathrm{~B}$ and ${ }^{11} \mathrm{~B}$ relaxation times, the activation energy for molecular reorientation was found to be $8.7 \pm 0.4 \mathrm{~kJ} / \mathrm{mol}(2.1 \pm 0.1 \mathrm{kcal} / \mathrm{mol})$. Consistent values for $\left|e^{2} q Q / h\right|$ were obtained from relaxation measurements in liquid phase and from c.w. spectra in solid phase. The temperature dependence of proton relaxation times deviates significantly from the activation energy model at higher temperatures, where spin-rotation interactions may be important. Proton transverse relaxation times $\left(T_{2}\right)$ have also been measured and are consistent with the AllerhandThiele theory.
\end{abstract}

Key words: Boron-10; boron-11; dimethoxyborane; proton; pulsed nuclear magnetic resonance; scalar and quadrupolar coupling constants and relaxation effects.

\section{Introduction}

In the past few years, several experimental wide-line NMR studies [1-3] ${ }^{1}$ of two-spin systems $\left(I=\frac{1}{2}, S>\frac{1}{2}\right)$ have been reported. The early experimental work $[1,2]$ was initiated in an attempt to determine metalhydrogen bond distances in transition-metal carbonyl hydrides. If one assumes, as everyone did at that time, that the Van Vleck moment theory [4] is valid for such systems, then in some cases metal-hydrogen bond distances can be obtained from the low-temperature proton wide-line NMR data. In studies of $\mathrm{HMn}(\mathrm{CO})_{5}$ and $\mathrm{HCo}(\mathrm{CO})_{4}$, values of 1.3 and $1.2 \mathrm{~A}$, respectively, were obtained for the metal-hydrogen bond distances. The subsequent observation in this laboratory that the proton line shapes for $\mathrm{HCo}(\mathrm{CO})_{4}$ and $\mathrm{H}^{10} \mathrm{~B}\left(\mathrm{OC}^{2} \mathrm{H}_{3}\right)_{2}$ are frequency dependent indicates that factors other than the direct dipole-dipole interaction can play an important role in determining the proton line shape and second moment of such systems. Since that time it has been shown theoretically $[3,5,6]$ that this is indeed the case. In this paper, we have examined the various interactions that may contribute to proton NMR line shapes and moments by

\footnotetext{
' Figures in brackets indicate the literature references at the end of this paper.
}

studying the systems $\mathrm{H}^{11} \mathrm{~B}\left(\mathrm{OC}^{2} \mathrm{H}_{3}\right)_{2}$ and $\mathrm{H}^{10} \mathrm{~B}\left(\mathrm{OC}^{2} \mathrm{H}_{3}\right)_{2}$ rather carefully. These molecules are not ideal, since the principal $z$-axis of the electric field gradient tensor does not coincide with the H-B internuclear vector. Consequently, detailed line shape calculations are not possible. Nevertheless, they do afford us the opportunity to determine some of the NMR and NQR parameters by at least two independent techniques. In this way, one can assess experimentally the accuracy of some of the newer pulse techniques to determine such parameters as spin-spin coupling constants $J_{i j}$ and electric quadrupole coupling constants $e^{2} q Q / h$ (in liquid samples), and one can check the validity of the approximations made in the analysis of two-spin systems and some of the predictions made by the theory. If these new pulse techniques can be shown to be valid and reliable, they can then be used with confidence to determine, for example, $J_{\mathrm{H}-\mathrm{Mn}}$ in $\mathrm{HMn}(\mathrm{CO})_{5}$; in such two spin $\left(I=\frac{1}{2}, S>\frac{1}{2}\right)$ systems, conventional c.w. techniques (high resolution and wide-line NMR) give no information about the coupling constants.'In this paper, we would like to call attention to the various interactions which can contribute to the proton NMR line shapes and moments, to show how the magnitudes of these interactions can be determined experimentally using pulsed and wide-line NMR techniques and to demonstrate the validity of these techniques. 


\section{Theory}

The second moment $\mathrm{M}_{2}$ of the proton NMR absorption spectrum in two-spin systems (e.g., $\mathrm{HMn}(\mathrm{CO})_{5}$, $\mathrm{HCo}(\mathrm{CO})_{4}, \mathrm{H}^{11} \mathrm{~B}\left(\mathrm{OC}^{2} \mathrm{H}_{3}\right)_{2}$, etc.) with spins $I=\frac{1}{2}$ and $S>\frac{1}{2}$ arises from several sources $[1,2,7]$ :

(a) The direct dipole-dipole interactions (Van Vleck's result [4]). For a two spin system, this is given by:

$$
M_{2}^{d-d}=(4 / 15) \hbar^{2} \gamma_{s}^{2} S(S+1) r^{-6}
$$

(b) The chemical-shift anisotropy interaction. This will be small for protons and may be neglected.

(c) Indirect dipole-dipole interaction. For a two-spin system $I=\frac{1}{2}$ and $S>\frac{1}{2}$, the nuclear spin $I$ is quantized along the magnetic field $\mathrm{H}_{0}$. On the other hand, the direction of quantization of the spin $S$ lies between $\mathrm{H}_{0}$ and the principal $z$ axis of the electric field gradient tensor. For convenience, we define the parameter

$$
\alpha=\left|4 S(2 S-1) \gamma_{s} \hbar \mathrm{H}_{0} /\left(e^{2} q Q\right)\right| .
$$

In the limit $\alpha \ll 1$, the spin $S$ is quantized along the $z$ axis of the field gradient tensor and for $\alpha \gg 1, \mathrm{~S}$ is quantized along $\mathrm{H}_{0}$. As has been pointed out $[3,6]$, both the line shape shape and second moment for the nucleus I change considerably in the region $\alpha \approx 4$. The direct (Van Vleck) interaction limit corresponds to $\alpha=\infty$.

(d) The spin spin coupling interaction. The contribution to $\mathrm{M}_{2}$ is given [8] by

$$
\mathrm{M}_{2}^{\text {s.c. }}=\frac{\mathrm{S}(\mathrm{S}+1)}{3} J^{2} .
$$

For $\mathrm{H}^{11} \mathrm{~B}\left(\mathrm{OCD}_{3}\right)_{2}$ where the ${ }^{11} \mathrm{~B}-\mathrm{H}$ spin-spin coupling constant $J_{\mathrm{H}-\mathrm{B}}=164 \mathrm{~Hz}$, the contribution to the second moment is very small. $J_{\mathrm{H}-\mathrm{Mn}}$ in $\mathrm{HMn}(\mathrm{CO})_{5}$ and $J_{\mathrm{H}-\mathrm{Co}}$ in $\mathrm{HCo}(\mathrm{CO})_{4}$ are unknown, but could be rather large. In order to be significant, $J$ would have to be greater than $1 \mathrm{kHz}$. Similarly, the contribution to $\mathrm{M}_{2}$ from the anisotropic part of spin-spin coupling is also very small [6].

In 2-spin systems where the fine-structure has been partially or completely wiped out because of rapid quadrupole relaxation of one of the nuclei, the spin-spin coupling constant $J_{\text {IS }}$ can, in principle still be obtained by pulsed NMR measurements. Following Abragam's notation [8], the relaxation rates, $R_{1}\left(R_{1}=1 / T_{1}\right)$ and $R_{2}$ of the nucleus $\mathrm{I}(\mathrm{I}=1 / 2, \mathrm{~S}>1 / 2)$ for dipolar relaxation are given by:

$$
\begin{aligned}
R_{2}^{\mathrm{I}}= & R_{1}^{\mathrm{I}}=\gamma_{\mathrm{I}}^{2} \gamma_{\mathrm{S}}^{2} \hbar^{2} \mathrm{~S}(\mathrm{~S}+1) \\
& \left\{\frac{1}{12} J^{(0)}\left(\omega_{\mathrm{I}}-\omega_{\mathrm{S}}\right)+\frac{3}{2} J^{(1)}\left(\omega_{\mathrm{I}}\right)+\frac{3}{4} J^{(2)}\left(\omega_{\mathrm{I}}+\omega_{\mathrm{S}}\right)\right\}
\end{aligned}
$$

For ${ }^{1} \mathrm{H}=\mathrm{I},{ }^{11} \mathrm{~B}=\mathrm{S}$ and $\omega \tau_{c} \ll 1$, we get:

$$
R_{2}^{\mathrm{I}}=R_{1}^{\mathrm{I}}=\frac{5 \gamma_{\mathrm{H}}^{2} \gamma_{\mathrm{B}}^{2} \hbar^{2}}{r_{\mathrm{H}-\mathrm{B}}^{6}} \tau_{c} \equiv \mathrm{DD}
$$

where $\gamma_{\mathrm{H}}$ and $\gamma_{\mathrm{B}}$ are the magnetogyric ratios of ${ }^{1} \mathrm{H}$ and ${ }^{11} \mathrm{~B}, r_{\mathrm{H}-\mathrm{B}}=1.25 \times 10^{-8} \mathrm{~cm}$, is the $\mathrm{H}-\mathrm{B}$ bond distance [7], $\tau_{c}$ is the correlation time for the isotropic reorientation of the molecule.

The proton relaxation times arising from the indirect scalar coupling interactions are given by [8]

$$
\begin{aligned}
& R_{1}^{\mathrm{I}}=\frac{2 A^{2}}{3} S(S+1) \frac{\tau_{s}}{1+\left(\omega_{\mathrm{I}}-\omega_{\mathrm{S}}\right)^{2} \tau_{s}^{2}} \\
& R_{2}^{\mathrm{I}}=\frac{A^{2}}{3} S(S+1)\left\{\frac{\tau_{s}}{1+\left(\omega_{\mathrm{I}}-\omega_{\mathrm{S}}\right)^{2} \tau_{s}^{2}}+\tau_{s}\right\}
\end{aligned}
$$

where $A$ is the spin-spin coupling constants in radians/s $(2 \pi J=A)$, and $\tau_{s}$ in this case is just the relaxation time of the nucleus $\mathrm{S}\left(R_{1}^{\mathrm{S}}=R_{2}^{\mathrm{S}}=\tau_{s}^{-1}\right)$ which is dominated by quadrupole relaxation effects [8]:

$$
R_{1}^{\mathrm{S}}=R_{2}^{\mathrm{S}}=\frac{3}{40} \frac{2 S+3}{S^{2}(2 S-1)}\left(1+\frac{\eta^{2}}{3}\right)\left(\frac{e q Q}{\hbar}\right)^{2} \tau_{c}
$$

The total proton (or nucleus I) relaxation times then are given by

$$
\begin{array}{r}
R_{1}^{\mathrm{I}}=\mathrm{DD}+\frac{2 A^{2}}{3} S(S+1) \frac{\tau_{s}}{1+\left(\omega_{\mathrm{I}}-\omega_{\mathrm{S}}\right)^{2} \tau_{s}^{2}} \\
R_{2}^{\mathrm{I}}=\mathrm{DD}+\frac{A^{2}}{3} S(S+1)\left[\frac{\tau_{s}}{1+\left(\omega_{\mathrm{I}}-\omega_{\mathrm{S}}\right)^{2} \tau_{s}^{2}}+\tau_{s}\right] .
\end{array}
$$

For our experiments, $\omega \sim 10^{8} \mathrm{~s}^{-1}, \tau_{s} \sim 10^{-4} \mathrm{~s}$, hence

$$
R_{1}^{\mathrm{I}}=\mathrm{DD}
$$

$R_{2}^{\mathrm{I}}-R_{1}^{\mathrm{I}}=\frac{1}{3} A^{2} S(S+1) \tau_{s}=\frac{1}{3} A^{2} S(S+1)\left(R_{1}^{S}\right)^{-1}$.

For very fast quadrupole relaxation of nucleus $S$ (e.g., $\mathrm{Mn}(\mathrm{CO})_{5}$ where $T_{1}^{M n} \approx 90 \mu \mathrm{s}$ ), a simple spin-echo experiment will give an accurate value of $R_{2}^{1}$. For slower quadrupole relaxation (e.g., $\mathrm{H}^{11} \mathrm{~B}\left(\mathrm{OCD}_{3}\right)_{2}$ where $\left.T_{1} B \approx 3 \mathrm{~ms}\right)$, Carr-Purcell experiments are required to overcome the residual spin-spin coupling interaction.

The spin-spin coupling constant, $J$, then is given by:

$$
2 \pi J=\left[\frac{3\left(R_{2}^{\mathrm{I}}-R_{1}^{\mathrm{I}}\right)}{S(S+1) R_{1}^{\mathrm{S}}}\right]^{1 / 2} .
$$

To obtain $e^{2} q Q / \hbar$ is also relatively easy. If we know the I-S bond distance and $R_{1}^{\mathrm{I}}$, then from eq (4) we obtained $\tau_{c}$. From the value of $\tau_{c}$ and $R_{1}^{\mathrm{S}}$ we can obtain $e^{2} q Q / \hbar$ from eq (7) (in most of the simple cases with which we are concerned the asymmetry parameters, $\eta$, is approximately zero).

\section{Experimental Procedure}

The synthesis [10] of the molecules $\mathrm{H}^{11} \mathrm{~B}\left(\mathrm{OC}^{2} \mathrm{H}_{3}\right)_{2}$ and $\mathrm{H}^{10} \mathrm{~B}\left(\mathrm{OC}^{2} \mathrm{H}_{3}\right)_{2}$ and a preliminary NMR study [7] 
have already been reported. The proton and ${ }^{11} \mathrm{~B}$ c.w. line shape studies were performed using a modified marginal oscillator [11], a gas-flow cryostat and a commercial magnet, magnet power supply and field sweep system. The field sweep was calibrated by the audio side-band technique. The pulsed NMR experiments were done using a spectrometer designed and built in this laboratory. The $\pi / 2$ pulse-widths were about $1 \mu \mathrm{s}$ in duration for protons and the overall system recovery time was about $16 \mu \mathrm{s}$. The $T_{1}$ values at each temperature were determined via the $\pi-\pi / 2$ pulse sequence; a least square fit of the data to the equation

$$
\ln [\mathrm{I}(\infty)-\mathrm{I}(t)]=\left(-t / T_{1}\right)+\mathrm{K}
$$

was used to obtain $T_{1}$. At least ten data points were used to obtain each $T_{1}$ value. A rms deviation of about 3 percent was typical for the $T_{1}$ values. The temperature of the gas-flow cryostat system was controlled by a servo-device which employed a platinum resistor as a sensing element. The temperature could be held constant to about $0.01{ }^{\circ} \mathrm{C}$. The sample temperature was measured with a copper-constantan thermocouple placed near the sample coil. Since the samples were synthesized in a high vacuum system the presence of even minute amounts of dissolved oxygen can be discounted. The final samples for our experiments were distilled in the high-vacuum system into $10 \mathrm{~mm}$ quartz sample tubes and sealed off. (Borosilicate glass containers are not suitable in this case because of the intense ${ }^{11} \mathrm{~B}$ resonance from the glass.)

\section{Results and Discussion}

\subsection{Line-Shape Studies}

Proton NMR spectra of $\mathrm{H}^{11} \mathrm{~B}\left(\mathrm{OCD}_{3}\right)_{2}$ were recorded from $9.7 \mathrm{MHz}(0.23 T=2.3 \mathrm{kG})$ to $53 \mathrm{MHz}$. All of them are quite similar to the previously recorded spectra [7] at $30 \mathrm{MHz}$. This reflects the fact that even at
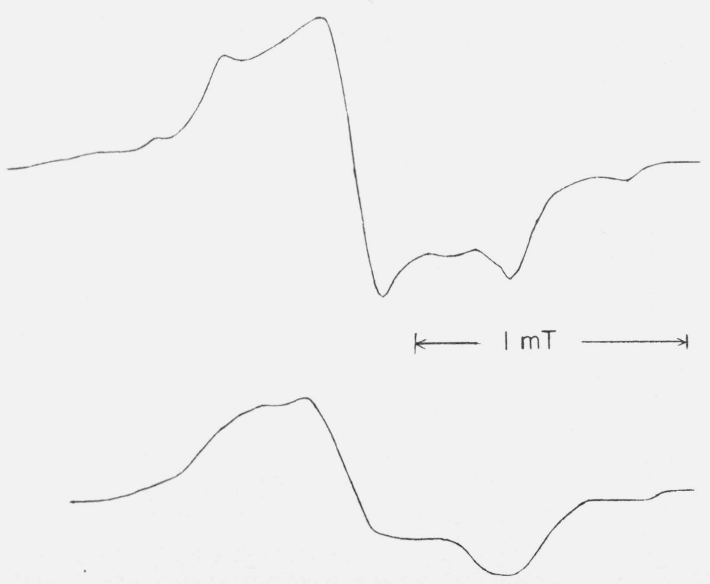

Figure 1. Proton absorption derivative spectra in solid $\mathrm{H}^{10} \mathrm{~B}\left(\mathrm{OCD}_{3}\right)_{2}$. At 52.8 (top) and 9.8 (bottom) $\mathrm{MHz}$.
$0.23 T, \alpha \cong 14$ and consequently no appreciable indirect dipolar interaction is to be expected.

For the $\mathrm{H}^{10} \mathrm{~B}\left(\mathrm{OCD}_{3}\right)_{2}$ molecule, the situation is quite different. Although the details are obscured due to the larger spin quantum number $\left(S=3\right.$ for $\left.{ }^{10} \mathrm{~B}\right)$ and the smaller magnetogyric ratio $\gamma\left({ }^{11} \mathrm{~B}\right) / \gamma\left({ }^{10} \mathrm{~B}\right) \cong 3$, there is a considerable difference in the proton spectra recorded at 10 and at $53 \mathrm{MHz}$; these spectra are shown in figure 1 . The predicted trends $[3,6]$ and the observed spectra are in good general agreement. A detailed comparison of the theory and the experimental results is not possible since the magnetic dipole-dipole vector does not coincide with the principal axis of electric field gradient and the calculations are therefore intractable.

The quadrupolar coupling constant $e^{2} q Q / h$ for boron-11 may be determined directly from the NMR spectra in the solid phase [12]. The spectra at 18.8, 12.7 , and $9.7 \mathrm{MHz}$ are shown in figure 2. Only the

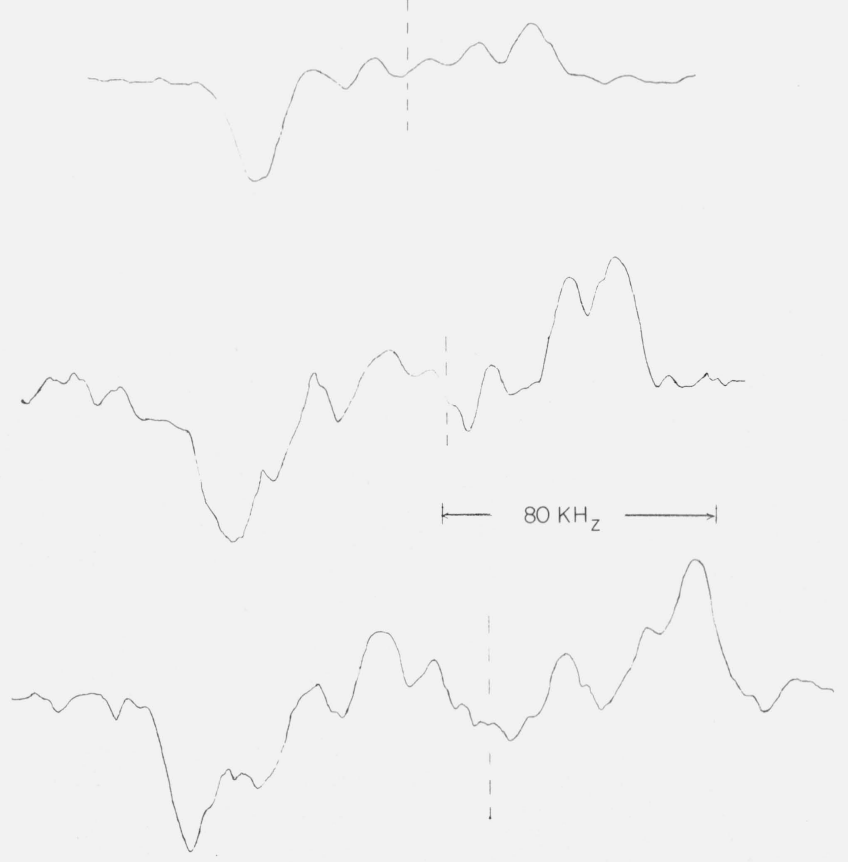

FIGURE 2. ${ }^{11} \mathrm{~B}$ spectra, absorption derivative, $\mathrm{HB}\left(\mathrm{OCD}_{3}\right)_{2}$ in quartz tube.

Top to bottom, 18.8, 12.7 and $9.7 \mathrm{MHz}$. Dashed lines indicate unshifted "1B resonance position. Magnetic field decreases from left to right.

central $\left(-\frac{1}{2} \rightarrow \frac{1}{2}\right)$ component, broadened by second order quadrupole splitting, can be seen. The spectra are in general agreement with the calculated line shape for a symmetric field gradient, indicating $\eta \ll 1$. However, fine structures due to the magnetic dipole interactions between ${ }^{11} \mathrm{~B}$ and proton nuclei are also observed. Including the magnetic dipolar contributions, the total splitting (in $\mathrm{Hz}$ ) is $(25 / 192)\left(e^{2} q Q / h\right)^{2} \nu_{\mathrm{L}}^{-1}+(\xi / h)$, where $\nu_{\mathrm{L}}$ is the rf oscillator frequency, $\xi=\gamma_{\mathrm{B}} \gamma_{\mathrm{H}} \hbar^{2} r^{-3}$, $\xi / h=20 \mathrm{KHz}$. The dipolar contribution, $\xi / h$, is about 10 percent of the total splitting. From figure 2, we get $\left|e^{2} q Q / h\right|=3.0 \pm 0.2 \mathrm{MHz}$ for ${ }^{11} \mathrm{~B}$ in the solid phase of $\mathrm{H}^{11} \mathrm{~B}\left(\mathrm{OCD}_{3}\right)_{2}$. (In this paper, \pm refers to rms deviations). 
Slightly lower values $\left(\left|e^{2} q Q / h\right|=2.5 \mathrm{MHz}\right)$ have been reported [13] for ${ }^{11} \mathrm{~B}$ coupling constants at triangularly coordinated sites in borates.

\subsection{Relaxation Studies}

Spin-lattice relaxation time $\left(T_{1}\right)$ data at various temperatures in the liquid phase are summarized in figure 3 . The proton relaxation times $T_{1 \mathrm{H}}$ at 4.2 and 19

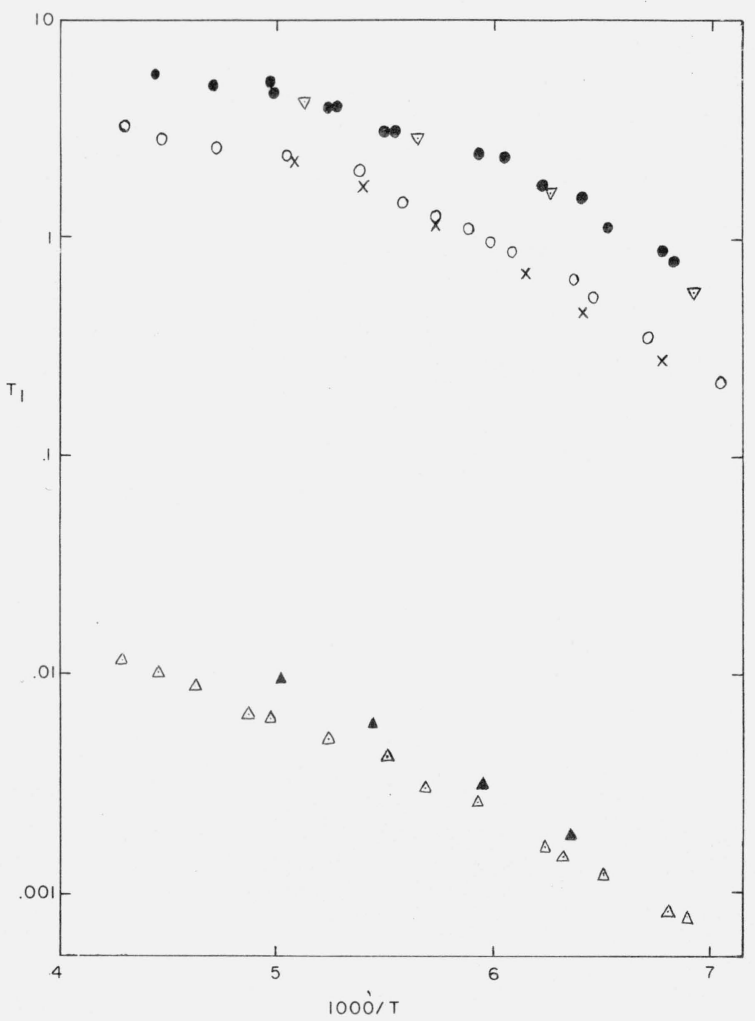

FIGURE 3. Spin-lattice relaxation times $\mathrm{T}_{1}$ (in s) versus inverse temperature on semilogarithm scale.

$\mathrm{H}^{11} \mathrm{~B}\left(\mathrm{OCD}_{3}\right)_{2}$ : proton at $19 \mathrm{MHz}, \bigcirc$; proton at $4.2 \mathrm{MHz}, \mathrm{x} ;{ }^{11} \mathrm{~B}$ at $19 \mathrm{MHz}, \triangle . \mathrm{H}^{10} \mathrm{~B}\left(\mathrm{OCD}_{3}\right)_{2}$ : proton at $19 \mathrm{MHz}, 0$; proton at $4.2 \mathrm{MHz}, \nabla ;{ }^{10} \mathrm{~B}$ at $4.2 \mathrm{MHz}, \mathbf{M}$.

$\mathrm{MHz}$ are not very different. Also, we observe that $T_{1 \mathrm{H}}$ for $\mathrm{H}^{10} \mathrm{~B}\left(\mathrm{OCD}_{3}\right)_{2}$ is a factor of $2.5 \pm 0.3$ longer than $T_{1 \mathrm{H}}$ for $\mathrm{H}^{11} \mathrm{~B}\left(\mathrm{OCD}_{3}\right)_{2}$ over the experimental temperature range $140-240 \mathrm{~K}$, and that the ${ }^{10} \mathrm{~B}$ relaxation time $T_{1 \mathrm{~B}-10}$ in $\mathrm{H}^{10} \mathrm{~B}\left(\mathrm{OCD}_{3}\right)_{2}$ is a factor of $1.4 \pm 0.2$ longer than the ${ }^{11} \mathrm{~B}$ relaxation time $\pi_{1 \mathrm{~B}-11}$ in $\mathrm{H}^{11} \mathrm{~B}\left(\mathrm{OCD}_{3}\right)_{2}$.

We shall consider the nuclear magnetic relaxation due to molecular rotation only, the rotational correlation time $\tau_{c}$ being very short $\left(\tau_{c} \ll 10^{-8} \mathrm{sec}\right)$. Then the proton relaxation time $T_{1 \mathrm{H}}$ due to $\mathrm{H}-\mathrm{B}$ dipolar interaction and the boron relaxation time due to electric quadrupole interaction are given by [8]:

$$
\begin{array}{r}
\left(T_{1 \mathrm{H}}\right)^{-1}=\frac{4}{3} \gamma_{\mathrm{B}}^{2} \hbar^{2} r^{-6} S(S+1) \tau_{c} \\
\left(T_{1 \mathrm{~B}}\right)^{-1}=\frac{3(2 S+3)}{40 S^{2}(2 S-1)}\left(\frac{e^{2} q Q}{\hbar}\right)^{2}\left(1+\frac{\eta^{2}}{3}\right) \tau_{c}
\end{array}
$$

where $\gamma_{\mathrm{B}}, S, e^{2} q Q / h$ and $\eta$ are the magnetogyric ratio, spin, quadrupolar coupling constant and asymmetry parameter of the boron nucleus, and $r$ is the H-B distance. From (15), $T_{1}$ for ${ }^{10} \mathrm{~B}$ is expected to be a factor of 1.54 longer than $T_{1}$ for ${ }^{11} \mathrm{~B}$, this ratio being independent of $q, \eta$ and $\tau_{c}$; experimentally, we find the ratio to be $1.4 \pm 0.2$. From (14), we expect $T_{1 \mathrm{H}}$ in $\mathrm{H}^{10} \mathrm{~B}\left(\mathrm{OCD}_{3}\right)_{2}$ to be a factor of 2.78 longer than $T_{1 \mathrm{H}}$ in $\mathrm{H}^{11} \mathrm{~B}\left(\mathrm{OCD}_{3}\right)_{2}$, in reasonable agreement with the ratio $2.5 \pm 0.3$ observed experimentally.

Since $\eta^{2} \ll 1$, the quadrupolar coupling constant $e^{2} q Q / h$ may be calculated from the ratio $T_{1 \mathrm{H}} / T_{1 \mathrm{~B}}$ which is independent of $\tau_{c}$. In $\mathrm{H}^{11} \mathrm{~B}\left(\mathrm{OCD}_{3}\right)_{2}$, we find $T_{1 \mathrm{H} i} T_{1 \mathrm{~B}}=360 \pm 40$ over our experimental temperature range in figure $3, T_{1 \mathrm{~B}}$ being the ${ }^{11} \mathrm{~B}$ relaxation time. Using $\mathrm{B}-\mathrm{H}$ bond distance $r=1.25 \mathrm{~A}$ from the proton c.w. resonance data [8], we get $e^{2} q Q / h=2.7$ $\pm 0.2 \mathrm{MHz}$ for ${ }^{11} \mathrm{~B}$ in $\mathrm{H}^{11} \mathrm{~B}\left(\mathrm{OCD}_{3}\right)_{2}$ from (14) and (15). This calculated value is expected to be slightly lower than the true ${ }^{11} \mathrm{~B}$ quadrupolar coupling constant, since we have considered only the intra molecular rotational ${ }^{11} \mathrm{~B}-\mathrm{H}$ dipolar relaxation for protons in (14). From the second order quadrupolar splitting in polycrystalline solids, we found $e^{2} q Q / h=3.0 \pm 0.2 \mathrm{MHz}$. A previous estimate of $2.6 \mathrm{MHz}$ has been given by Boden, Gutowsky, Hansen, and Farrar [9] from pulse measurements in liquids.

Rotational correlation times for liquids $\mathrm{HB}\left(\mathrm{OCD}_{3}\right)_{2}$ from $T_{1}$ data using (14) and (15) are summarized in figure 4 , using the B-H bond distance $r=1.25 \mathrm{~A}$ and

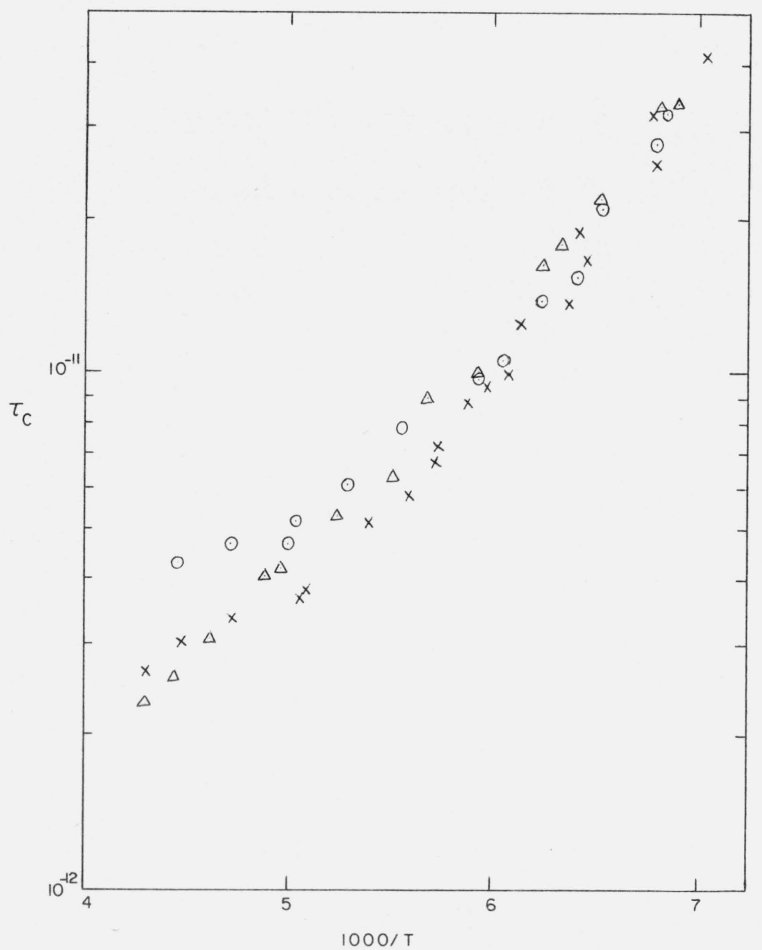

FIGURE 4. Rotational correlation time (in s) in liquid $\mathrm{HB}\left(\mathrm{OCD}_{3}\right)_{2}$ versus inverse temperature on semilogarithm scale: from proton data in $\mathrm{H}^{11} \mathrm{~B}\left(\mathrm{OCD}_{3}\right)_{2}$, $\mathrm{x}$; from proton data in $\mathrm{H}^{10} \mathrm{~B}\left(\mathrm{OCD}_{3}\right)_{2}, \odot$; from ${ }^{11} \mathrm{~B}$ data in $\mathrm{H}^{11} \mathrm{~B}\left(\mathrm{OC} \mathrm{D}_{3}\right)_{2}, \triangle$. 
$e^{2} q Q / h=2.7 \mathrm{MHz}$ for ${ }^{11} \mathrm{~B}$. The agreement between various methods is satisfactory, indicating that (14) and (15) are in general correct, although the problem is somewhat oversimplified. We expect the boron nuclear relaxation to be dominated by quadrupolar interaction. The correlation times from the boron relaxation can be fitted to usual form:

$$
\tau_{c}=\tau_{0} e^{V / R T}
$$

where $V$ is the activation energy, with $V=(8.7 \pm 0.4)$ $\times 10^{3} \mathrm{~J} / \mathrm{mol}(2.1 \pm 0.1 \mathrm{kcal} / \mathrm{mol})$. Previous studies [9] over a narrower temperature range gave a somewhat lower activation energy, $(5.5 \pm 0.4) \times 10^{3} \mathrm{~J} / \mathrm{mol}$.

On the other hand, the proton relaxation times in $\mathrm{H}^{11} \mathrm{~B}\left(\mathrm{OCD}_{3}\right)_{2}$ and $\mathrm{H}^{10} \mathrm{~B}\left(\mathrm{OCD}_{3}\right)_{2}$ cannot be fitted to the activation energy from (16); whereas the fit is reasonably good in the low temperature region, the $T_{1}$ versus $1 / T$ curve is considerably flatter than expected in the $10^{3} / T>5$ region. Similar behaviors have been reported, for example, by Powles and co-workers [14], and their results have been interpreted through spinrotation interactions. This may well be the cause of

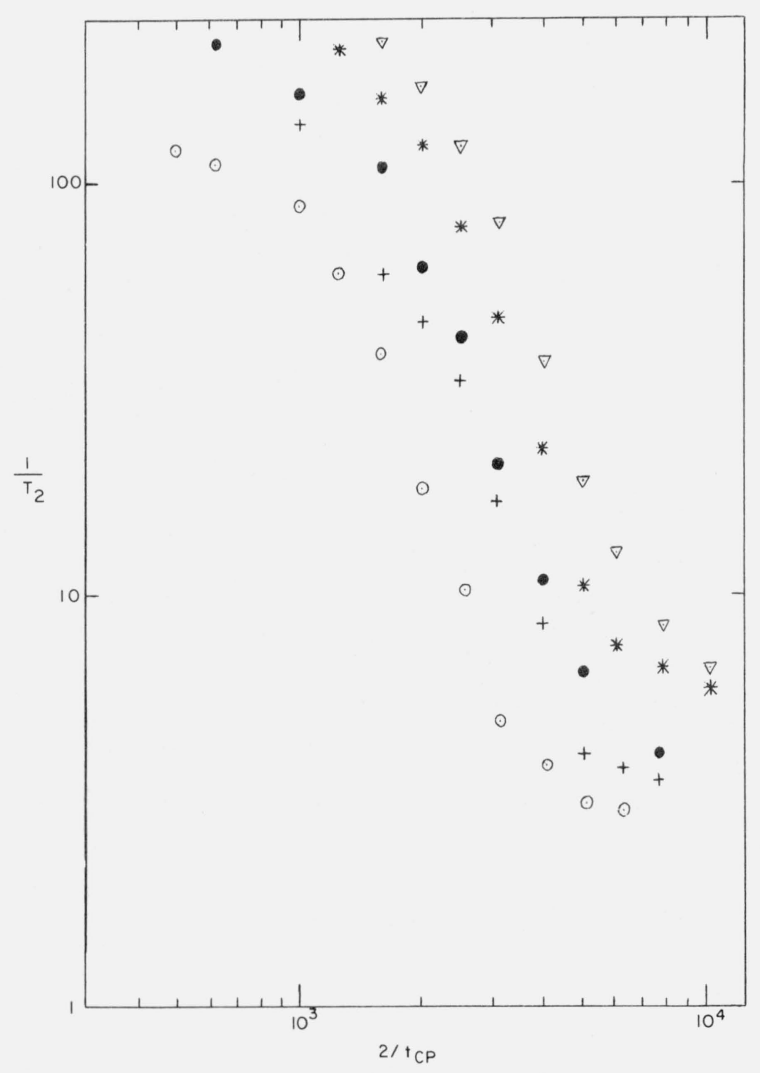

FiguRE 5. $1 / \mathrm{T}_{2}$ versus $1 / \mathrm{t}_{\mathrm{cp}}$ for proton in $\mathrm{H}^{11} \mathrm{~B}\left(\mathrm{OCD}_{3}\right)_{2}$. $198 \mathrm{~K}, \odot ; 175 \mathrm{~K},+: 167 \mathrm{~K}, \bullet: \mathrm{F} 55 \mathrm{~K},{ }^{*} ; 147 \mathrm{~K}, \nabla$. the nonlinear high-temperature dependence of $T_{1 \mathrm{H}}$ versus temperature for $\mathrm{H}^{11} \mathrm{~B}\left(\mathrm{OCD}_{3}\right)_{2}$.

Transverse proton relaxation times $T_{2 \mathrm{H}}$ have been measured by the Carr-Purcell pulse sequence in the liquid phase of $\mathrm{H}^{11} \mathrm{~B}\left(\mathrm{OCD}_{3}\right)_{2}$, and the results are summarized in figure 5 . At $147 \mathrm{~K}$, our results are in good agreement with the fast quadrupolar relaxation limit [15] when $\tau_{c p} \geqq T_{1 \mathrm{~S}}$ :

$$
\begin{array}{r}
\left(T_{2 \mathrm{H}}\right)^{-1}=\left(T_{1 \mathrm{H}}\right)^{-1}+\frac{1}{3} S(S+1) A^{2} T_{1 \mathrm{~S}}\left(2 T_{1 \mathrm{~S}} / t_{c p}\right) \\
\left.\tanh \left(t_{c p} / 2 T_{1 \mathrm{~S}}\right)\right]
\end{array}
$$

where $S$ refers to ${ }^{11} \mathrm{~B}, T_{1 \mathrm{~S}}=6.2 \times 10^{-4} \mathrm{sec}$. and $T_{1} \mathrm{H}$ $=0.2$ sec. from figure $3, A=2 \pi J$. From our data, we estimate the ${ }^{11} \mathrm{~B}-\mathrm{H}$ spin-spin coupling constant $J$ $=170 \mathrm{~Hz}$, in agreement with the previous high resolution studies [9].

In conclusion, we believe that these results demonstrate that pulsed NMR techniques can, in favorable cases be used to obtain reliable, reasonably accurate values for spin-spin and quadrupole coupling constants in liquids.

\section{References}

[1] Farrar, T. C., Ryan, W., Davison, A., and Faller, J. W., J. Am. Chem Soc. 88, 184 (1966).

[2] Farrar, T. C., Brinckman, F. E., Coyle, T. D., Davison, A., and Faller, J. W., Inorg. Chem. 6, 161 (1967).

[3] Vander Hart, D. L., Gutowsky, H. S., and Farrar, T. C., J. Am. Chem. Soc. 89, 5056 (1967).

[4] Van Vleck, J. H., Phys. Rev. 74, 1168 (1948).

[5] Sheldrick, G. M., Chem. Comm., 751 (1967); Mol. Phys. 13, 399 (1967).

[6] Vander Hart, D. L., and Gutowsky, H. S., J. Chem. Phys. 49, 261 (1968); Vander Hart, D. L., Gutowsky, H. S., and Farrar, T. C., J. Chem. Phys. (to be published, 1969).

[7] Farrar, T. C., Cooper, J., and Coyle, T. D., Chem. Comm. 610 (1966).

[8] Abragam, A., The Principles of Nuclear Magnetism (Clarendon Press, London (1961), pp. 294, 299,311,314, 331 and 438; his notation will be followed here in general.

[9] Boden, N., Gutowsky, H. S., Hansen, J. R., and Farrar, T. C., J. Chem. Phys. 46, 2849 (1967).

[10] Burg, A. B., and Schlesinger, H. I., J. Am. Chem. Soc. 55, 4020 (1933).

[11] Gutowsky, H. S., Meyer, L. H., and McClure, R. E., Rev. Sci. Instr. 24, 644 (1953).

[12] Cohen, M. H., and Reif, F., Solid State Physics 5, 339 (1957); their notation will be followed here in general.

[13] Cuthbert, J. D., MacFarlane, W. T., and Petch, H. E., J. Chem. Phys. 43, 173 (1965); Lal, K. C., and Petch, H. E., J. Chem. Phys. 43, 178 (1965).

[14] Powles, J. G., and Figgins, R., Molecular Physics 13, 253 (1967); Green, D. K., and Powles, J. G., Proc. Phys. Soc. (London) 85, 87 (1965); Aksnes, D. W., Rhodes, M., and Powles, J. G., Molecular Physics 14, 333 (1968).

[15] Allerhand, A., and Thiele, E., J. Chem. Phys. 45, 902 (1966); Alger, T. D., and Gutowsky, H. S., J. Chem. Phys. 48, 4625 (1968).

(Paper 73A2-544) 